\title{
Reliability assessment of power distribution systems using disjoint path-set algorithm
}

\author{
Abdrabbi Bourezg $\cdot$ H. Meglouli
}

Received: 30 July 2013/Accepted: 23 July 2014/Published online: 22 October 2014

(C) The Author(s) 2014. This article is published with open access at Springerlink.com

\begin{abstract}
Finding the reliability expression of different substation configurations can help design a distribution system with the best overall reliability. This paper presents a computerized and implemented algorithm, based on Disjoint Sum of Product (DSOP) algorithm. The algorithm was synthesized and applied for the first time to the determination of reliability expression of a substation to determine reliability indices and costs of different substation arrangements. It deals with the implementation and synthesis of a new designed algorithm for DSOP implemented using $\mathrm{C} / \mathrm{C}++$, incorporating parallel problem solving capability and overcoming the disadvantage of Monte Carlo simulation which is the lengthy computational time to achieve satisfactory statistical convergence of reliability index values. The major highlight of this research being that the time consuming procedures of the DSOP solution generated for different substation arrangements using the proposed method is found to be significantly lower in comparison with the time consuming procedures of Monte Carlo-simulation solution or any other method used for the reliability evaluation of substations in the existing literature such as meta-heuristic and soft computing algorithms. This implementation gives the possibility of RBD simulation for different substation configurations in $\mathrm{C} / \mathrm{C}++$ using their path-set Boolean expressions mapped to probabilistic domain and result in simplest Sum of Disjoint Product which is on a one-to-one correspondence with reliability expression. This software
\end{abstract}

\footnotetext{
A. Bourezg $(\bowtie) \cdot$ H. Meglouli

Oil and Chemistry Faculty, University of Boumerdes,

Boumerdes, Algeria

e-mail: abourezg@yahoo.fr

H. Meglouli

e-mail: hmeglouli@yahoo.fr
}

tool is capable of handling and modeling a large, repairable system. Additionally, through its intuitive interface it can be easily used for industrial and commercial power systems. With simple Boolean expression for a configuration's RBD inputted, users can define a power system utilizing a RBD and, through a fast and efficient built-in simulation engine, the required reliability expressions and indices can be obtained. Two case studies will be analyzed in this paper. The effects of different substation configurations on the reliability are analyzed and compared. Then, the reliability of a radial distribution system will be evaluated using DSOP solution. The failure results will be combined with a load flow scenario, and indices such as SAIDI, SAIFI will be determined.

Keywords Boolean function - Disjoint product - Power substation reliability $\cdot \mathrm{RBD}$ and reliability indices

\section{Introduction}

Since distribution systems account for up to $90 \%$ of all customer reliability problems, improving distribution reliability is the key to improve customer reliability (Brown 2002).

Reliability evaluation of distribution power system is of significant importance when performing asset management. Distribution systems begin at distribution substations, which are the weakest link between the source of supply and the customer load points in a distribution power system, because they comprise switching arrangements that would lead to loss of load.

By knowing how to calculate the reliability of different substation configurations, an engineer can use this information to help design a system with the best overall 
reliability. But determining the reliability of a substation can also be important for existing installations as it can help locate weak points that may be contributing to overall system unreliability.

The reliability of substation must be high. However, once a reasonable level of reliability is achieved, there must be a means of evaluating the cost of potential changes to the substations to improve their reliabilities. Historically, the results of applying different reliability methodologies and tools varied significantly, and comparisons were difficult. The reliability analysis techniques working group of the Gold Book (IEEE Std. 493-1997) developed a standard network to enable comparison of analytical techniques. This paper describes the approach of simulations via reliability block diagrams as applied to the Gold Book standard network. Reliability indices of substations arrangements are presented, and are compared with each other.

The research indicated that users were utilizing a wide variety of tools and techniques with different analysis results. Furthermore, the only recommended methodology presented in IEEE Std 493 since 1980 was the "series and parallel" reliability methodology and the minimal cut-set method which estimated the frequency and duration of load point interruptions (IEEE Std 493-1997 1998).

The different approaches identified in (Hale et al. 2001) include:

- Zone branch;

- Reliability block diagram;

- Event tree;

- Monte Carlo (and discrete event simulations);

- Boolean algebra;

- FMECA;

- Cut-set.

These analytical approaches are applied to the IEEE Gold Book standard network in a series of papers to determine the accuracy of their results and how closely they can verify operational anomalies (Koval et al. 2002; Wang and Loman 2002).

Other approaches applicable to R/A analysis of industrial and commercial power systems are the following:

- Path-set;

- Fault tree;

- Markov Model;

- Petri nets.

This paper addresses the simulation approach as applied through a reliability block diagram (RBD).

The presented implementation is of a general-purpose algorithm for producing reliability expressions from reliability blocks diagrams. The algorithm is based on the transformation of the path-set expression (Boolean expression) derived from the reliability block diagram (RBD) into a sum of disjoint product. The final disjoint version of pathset can be interpreted directly as a probabilistic expression (system reliability) on a one-for-one correspondence. The input to this package would be the sum of the RBD path-sets and the output would be the system reliability expression. This algorithm could considerably reduce the number of disjoint (mutually exclusive) terms and save computation time with respect to top-event probability. Four major theorems of this algorithm are given, the use and correctness of which will be analyzed and proven. In addition, some examples for different substations configurations are illustrated and comparison of their reliability indices is provided to show the superiority and efficiency of the presented algorithm, which is not only easier to understand and implement but also better than the existing known SDP algorithm for large network and complex RBDs.

This implementation allows the analysis of the RBD as a time dependent analysis using $\mathrm{C} / \mathrm{C}++$. The contribution of this work is to provide a software tool for customers who purchase the critical power systems, the people who sell the systems, engineers who design and test the systems, and managers who make decisions on the systems. With knowledge of the system design (such as a one-line drawing), engineers can easily construct, verify, and modify the RBD, and also communicate with those of different disciplines. Reliability indices of different substations arrangements are presented, and are compared with each other to optimize the choice of the adequate configuration.

\section{Reliability evaluation and reliability indices}

There are two types of approaches or models that are used in reliability assessment, namely, non-state space based models (such as: network approach or fault trees) and state space based models (the most common of these is the continuous time Markov chain). Each of these approaches is used where its advantages are needed, and its disadvantages are harmless. An overview of these analytical methods is presented hereafter.

Network approach

In this approach, the topology of the network taken into consideration is represented in a logic block diagram (RBD). This diagram describes logical connections between components. Each block is a component which is removed when the component fails and replaced when it is repaired. The connections between the blocks describe the success or failure of the system as a function of the states of the component. Once the block diagram is settled, this approach can be handled in two ways. 


\section{Network reduction}

This method proceeds by the manipulation of the basic network structures: serial structures, parallel structures and $\mathrm{m} / \mathrm{n}$ structures when the $\mathrm{n}$ blocks originate from a common node.

The method sequentially reduces the simple structures to equivalent units until the whole network reduces to a single unit.

Series blocks are replaced with one block, where:

$A_{\mathrm{eq}}=\prod_{\forall i} A_{i}$ and $\lambda_{\mathrm{eq}}=\sum_{\forall i} \lambda_{i}$.

Parallel blocks and $\mathrm{m} / \mathrm{n}$ structures are replaced with one block where:

$U_{\mathrm{eq}}=\prod_{\forall i} U_{i} \quad$ and $\quad \mu_{\mathrm{eq}}=\sum_{\forall i} \mu_{i}$.

The above steps are repeated until the whole network reduces to an equivalent block. If at any stage the network does not reduce any further, the conditional probability theorem is used.

$$
\begin{aligned}
& P(\text { System success }) \\
& =P(\text { System success } \mid \text { key comp success }) \\
& \quad \times P(\text { key comp success }) \\
& \quad+P(\text { System success } \mid \text { key comp failure }) \\
& \quad \times P(\text { key comp failure })
\end{aligned}
$$

The network is decomposed into two networks; in the first, the key component is replaced with a short circuit (component success) and in the second, the key component is removed (component failure). The overall reliability of the network is as described by the previous formula.

When the block diagram is complex, decomposition into simple series and parallel paths may not be easy. The process could be quite difficult to program because it would require a lot of scanning. In this case, using cut sets or path-sets is way better.

\section{Path-set approach}

A path-set is a set of components whose functioning alone will guarantee system success. A minimal path-set has no subset of components whose functioning alone would ensure system success.

In the minimal path all the blocks constituting it are in series. The failure of any one of these blocks would render that ineffective. However, the minimal paths themselves are in parallel as the system will be successful as long as there is one path available between the input and output of the reliability block diagram RBD. The reliability of the network using this approach, and based on the inclusionexclusion theorem, is:

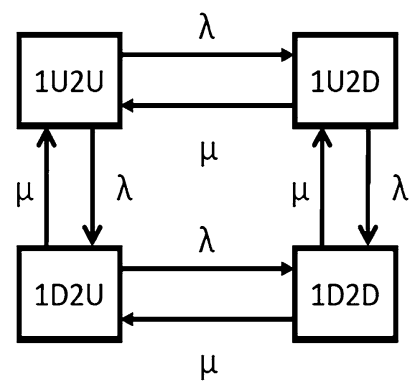

Fig. 1 State transition diagram of two identical components

$$
\begin{aligned}
P\left\{\bigcup_{r=1}^{n} M_{r}\right\}= & \sum_{r=1}^{n} P\left\{M_{r}\right\}-\sum_{1 \leq r<s \leq n} P\left\{M_{r} \cap M_{s}\right\} \\
& +\sum_{1 \leq r<s<t \leq n} P\left\{M_{r} \cap M_{s}\right\}+\cdots \\
& +(-1)^{n-1} P\left\{\bigcap_{r=1}^{n} M_{r}\right\}
\end{aligned}
$$

where $M_{i}$ s are the minimal path-sets. The total number of terms in this expression is $2^{n}-1$ where $n$ is the number of tie sets.

The network approach when applicable usually provides a shorter route to solution. The network approach is usually not suitable when dependent failures or repairs are involved (common cause failures, restricted repairs, warm standby unit, etc.). It is not necessary to assume the event independence in this approach, but dependent events can greatly increase the algebra of the computations.

State space approach

A component may assume various states depending upon its failure and restorative modes. The system state describes the states of the components and the environment in which the system is operating. The set of all the possible states of the system is called the state space or event space. If the environment can exist in $m$ states and the $n$ components of the system are independent in each environment state, then the state space consists of $2^{n+m}$ states. The number of states is, however, modified because of the dependency restrictions. The state space approach involves the following steps:

Indentify all possible states: describe all state space and transitions among states (Fig. 1).

Form transition rate matrix: this matrix is formed from the state vectors of the different components (using Kronecker product and Kronecker sum). This matrix is also known as transition matrix.

In case of dependency between components, the matrix is modified accordingly. The probability of every single 
state can be calculated by solving the Kolmogorov equations, written in matrix form hereafter:

$\left[\dot{P}_{i}(t)\right]=\left[\lambda_{i j}\right]\left[P_{i}(t)\right]$

where $\left[P_{i}(t)\right]$ is the probability vector of the $n$ states.

The reliability of the system is obtained by summing up states' probabilities. This approach is conceptually general and flexible and makes it possible to take into account various dependent failures (Dr Nahman 2002; Anderson 1998; Billinton and Allan 1996).

\section{Reliability indices for distribution power system}

The most common indices are SAIFI, SAIDI, CAIDI, and ASAI. SAIFI and SAIDI are system-oriented measures of frequency and duration of interruptions. CAIDI and ASAI are customer-oriented measures of outage duration (per outage) and fraction of demand satisfied. CAIDI and CAIFI are also important measures of outage duration and interruption frequency experienced by customers.

System average interruption duration index (SAIDI)

The most often used performance measurement for a sustained interruption is the system average interruption duration index (SAIDI). This index measures the total duration of an interruption for the average customer during a given time period. SAIDI is normally calculated on either monthly or yearly basis; however, it can also be calculated daily, or for any other time period (Brown 2002).

SAIDI $=\frac{\sum(\mathrm{ri} \times \mathrm{Ni})}{\mathrm{Nt}}$

Customer average interruption duration index (CAIDI)

Once an outage occurs the average time to restore service is found from the customer average interruption duration index (CAIDI). CAIDI is calculated similar to SAIDI except that the denominator is the number of customers interrupted versus the total number of utility customers (Brown 2002).

CAIDI $=\frac{\sum(\mathrm{ri} \times \mathrm{Ni})}{\mathrm{Ni}}$

System average interruption frequency index (SAIFI)

The system average interruption frequency index (SAIFI) is the average number of times that a system customer experiences an outage during the year (or time period under study). The SAIFI is found by dividing the total number of customers interrupted by the total number of customers served. SAIFI, which is a dimensionless number, is (Brown 2002):
$\mathrm{SAIFI}=\frac{\sum \mathrm{Ni}}{\mathrm{Nt}}$.

SAIFI can also be found by dividing the SAIDI value by the CAIDI value:

$\mathrm{SAIFI}=\frac{\text { SAIDI }}{\mathrm{CAIDI}}$.

Customer average interruption frequency index (CAIFI)

Similar to SAIFI is CAIFI, which is the customer average interruption frequency index. The CAIFI measures the average number of interruptions per customer interrupted per year. It is simply the number of interruptions that occurred divided by the number of customers affected by the interruptions. The CAIFI is (Brown 2002).

CAIFI $=\frac{\sum \mathrm{No}}{\mathrm{Ni}}$

Average service availability index (ASAI)

The average service availability index (ASAI) is the ratio of the total number of customer hours that service was available during a given time period to the total customer hours demanded. This is sometimes called the service reliability index. The ASAI is usually calculated on either a monthly basis $(730 \mathrm{~h})$ or a yearly basis $(8,760 \mathrm{~h})$, but can be calculated for any time period. The ASAI is found as (Brown 2002):

$\mathrm{ASAI}=\left(1-\frac{\sum(\mathrm{ri} \times \mathrm{Ni})}{\mathrm{Nt} \times T}\right) \times 100$

$T=$ Time period under study, hours.

$\mathrm{ri}=$ Restoration time, minutes.

$\mathrm{Ni}=$ Total number of customers interrupted.

$\mathrm{Nt}=$ Total number of customers served.

No $=$ Number of interruptions.

\section{Algorithm}

The evaluation of network reliability, with two state components, is a common task in power distribution systems' reliability assessment. And with an increase in networks' size and complexity, the computation workload is assigned to computers. However, applying the previously discussed methods will result in a NP-hard problem. Heuristic algorithms do not provide an assurance for optimization of the problem. These methods are an approximation (Bashiri and Karimi 2012). They have an additional property that worst-case solutions are known. Meanwhile, none of meta-heuristic algorithms are able to present a higher performance than others in solving all problems. Also, existing algorithms suffer from some 
drawbacks such as slow convergence rate, trapping into local optima, having complex operators, long computational time, need to tune many parameters and design for only real or binary search space. Hence, proposing new meta-heuristic algorithms to minimize the disadvantages is an open problem (Beheshti 2013). Also, Neuro computing and evolutionary computation usually need a lot of computational time, which is the disadvantage of the implementation of soft computing (Dote and Ovaska 2001).

State space approach: with $n$ components, the event space consists of $2^{n}$ states. The probability of each of the states is to be computed.

Fault trees: requires the use of cut-set or tie-set. These sets are to be disjoint, according to the probability expression that is used for limited mutual independent events, resulting in $2^{n}-1$ items.

$$
\begin{aligned}
P\left\{\bigcup_{r=1}^{n} M_{r}\right\}= & \sum_{r=1}^{n} P\left\{M_{r}\right\}-\sum_{1 \leq r<s \leq n} P\left\{M_{r} \cap M_{s}\right\} \\
& +\sum_{1 \leq r<s<t \leq n} P\left\{M_{r} \cap M_{s}\right\}+\cdots+ \\
& (-1)^{n-1} P\left\{\bigcap_{r=1}^{n} M r\right\}
\end{aligned}
$$

where $M_{i}$ s are the minimal sets.

Network approach: either use of network reduction method, which is impractical. Or path-set method that will lead to same result as fault tree approach.

To overcome the previous difficulties, an algorithm for calculating system reliability by sum of disjoint products (SDP), based on Boolean algebra, is presented. This algorithm is applied to sum of minimal path-sets (Xing 2012).

Sum of disjoint products algorithm

The first step to decompose a sum of product is rather simple. The recursive method can be used for example.

Assume that $M_{1}, M_{2}, M_{3} \ldots M_{n}$, are minimal path-sets, and $T$ is a sum of minimal path-sets, Then, using recursive method:

$$
\begin{aligned}
T & =\bigcup_{k=1}^{n} M_{k} \\
& =M_{1}+\overline{M_{1}} M_{2}+\overline{M_{1} M_{2}} M_{3}+\cdots+\overline{M_{1} M_{2}} \cdots \overline{M_{n-1}} M_{n}
\end{aligned}
$$

$T=F_{1}+F_{2}+F_{3}+\cdots+F_{n}$

where $F_{r}=C_{\mathrm{r}} M_{\mathrm{r}}$

and

$$
C_{r}=\left\{\begin{array}{cc}
1 & r=1 \\
C_{r-1} \overline{M_{r-1}} & 1<r \leq n
\end{array} .\right.
$$

Obviously, disjoint products between items of formula (2) can be achieved, but each item is crossed. As a result, we decompose the complement set of each path-set with De Morgan's law, and continue disjoint treatment. Since the number of basic event of applicable then the steps to calculate the sum of products' probability will be considerably reduced.

\section{Discipline for simplification}

The disciplines presented are based on Boolean algebra.

\section{Distinction discipline}

Supposing that minimal path-sets $M_{1}, M_{2}, M_{3} \ldots M_{k}$ have not the same basic events, it is not essential to decompose the product item $\overline{M_{1} M_{2}} \ldots \overline{M_{k-1}} M_{k}$ during quantitative calculation. If the probabilities are known as

$$
\begin{aligned}
& P_{M_{1}}, P_{M_{2}}, P_{M_{3}}, P_{M_{k-1}} \ldots P_{M_{k}} \text { then } \\
& P\left(\bar{M}_{1} M_{2} \ldots \overline{M_{k-1}} M_{k}\right)=\left(1-P_{M_{1}}\right)\left(1-P_{M_{2}}\right)\left(1-P_{M_{3}}\right) \ldots \\
& \ldots\left(1-P_{M_{k-1}}\right) P_{M_{k}}
\end{aligned}
$$

where

$P_{M_{k}}=\prod p_{j k}$ $k$.

$p_{j k}$ stands for the probability of the event $j$ in the path-set

\section{Elimination procedure}

If the minimal path-sets $M_{1}, M_{2}, M_{3} \ldots M_{k}$ have part event that are included in $M_{k}$, then these events can be eliminated from $M_{1}, M_{2}, M_{3} \ldots M_{k-1}$.

New sets are formed $M_{1 c}, M_{2 c}, M_{3 c}, \ldots M_{(k)}$

where

$\overline{M_{1} M_{2}} \ldots \overline{M_{k-1}} M_{k}=\overline{M_{1 c} M_{2 c}} \ldots \overline{M_{(k-1) c}} M_{k}$.

\section{Absorption discipline}

In the sets $M_{1 c}, M_{2 c}, M_{3 c}, \ldots M_{(k-1) c}$ that are dealt with elimination discipline, if the set $M_{i c}$ has all the events of $M_{j c}$ then $M_{i c}$ is absorbed by $M_{j c}$ so $\overline{M_{l c} M_{j c}} M_{k}=\overline{M_{j c}} M_{k}$.

\section{Decomposition discipline}

If $M_{1 c}, M_{2 c}, M_{3 c}, \ldots M_{(k-1) c}$, dealt with elimination discipline have a few same basic events, then we could use the formula below to decompose

$\overline{M_{l c} M_{j c}} M_{k}=\left(\overline{m_{l j c}}+M_{i j c} \overline{M_{l c c} M_{j c c}}\right) M_{k}$ 
where $M_{i c c}$ and $M_{j c c}$ stand for the products of basic events left except the same events of $M_{i c}$ and $M_{j c}$.

Computer program implementation

\section{Implementation procedure}

The first input to the program is the sum of minimal pathsets. Therefore, the first thing to do is decomposing it into a sum of mutually exclusive products. This step is accomplished using the recursive method.

Since each of the resulting products is crossed, then it is disjoint separately, using the four disciplines aforementioned.

First, the product is checked for distinction between the minimal path-sets' basic events. If the entire minimal pathsets (either complemented or not), forming this particular product, have independent events; therefore, the probability of this product can be computed straight forward. (Distinction discipline).

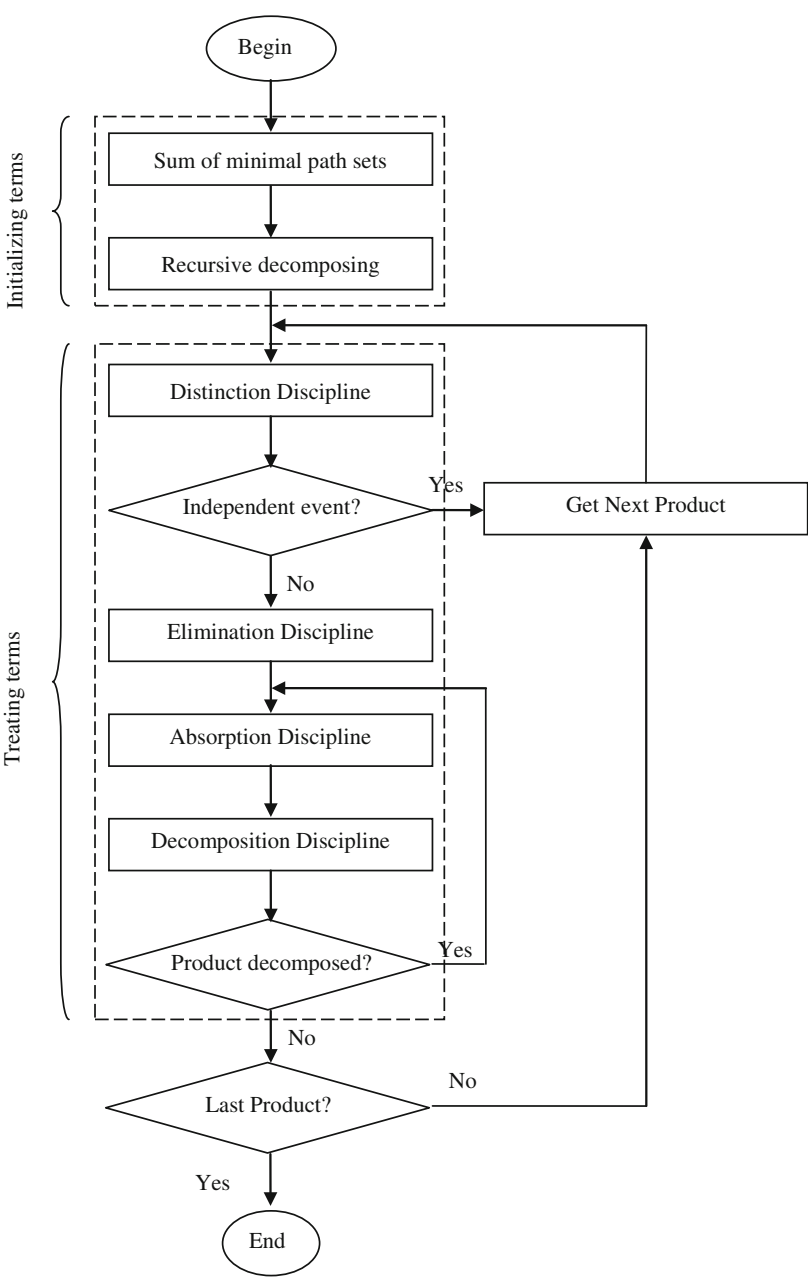

Fig. 2 Flow chart of the disjoint path-set algorithm
If the product is composed of dependent event then it is checked for repeated events in complemented path-sets and the uncomplemented one. If any then it is discarded. (Elimination discipline).

After the previous step, the complemented path-sets of the product are checked for inclusion between them. If a minimal path-set includes another one then it is absorbed by the latter (i.e. the first is discarded and the second is kept). (Absorption discipline).

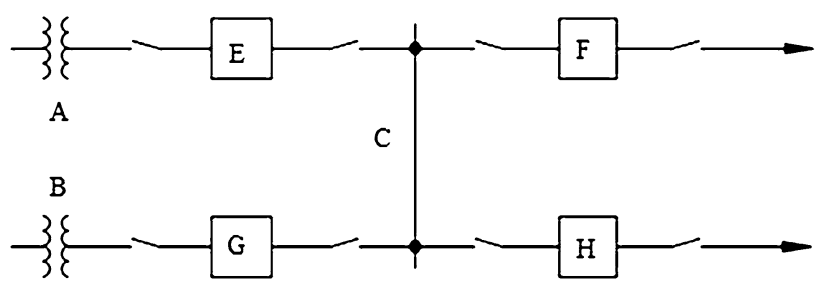

Fig. 3 Single bus with labeled components

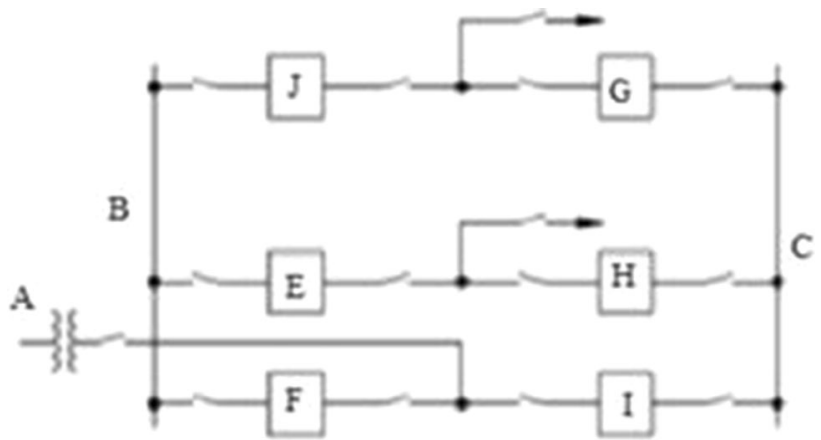

Fig. 4 Double breaker-double bus labeled components

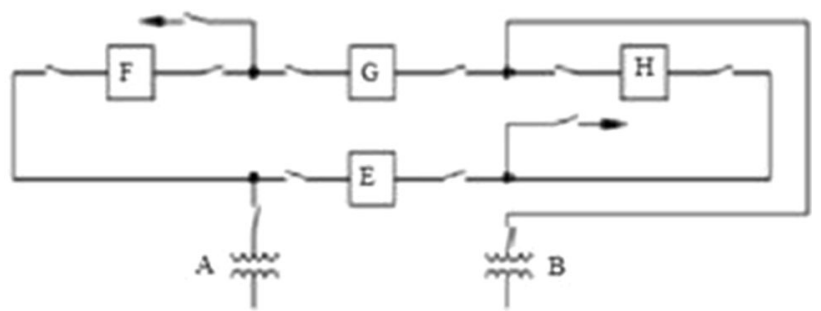

Fig. 5 Ring bus labeled components

Table 1 Substation component reliability data (IEEE Std 493-1997 1998)

\begin{tabular}{llrlr}
\hline Component & $\begin{array}{l}\text { Failure rate } \\
\text { (failure/year) }\end{array}$ & $\begin{array}{l}\text { MTTR } \\
(\mathrm{h})\end{array}$ & $\begin{array}{l}\text { Repair rate } \\
\text { (repair/year) }\end{array}$ & \multicolumn{1}{l}{$\begin{array}{l}\text { Cost } \\
(\$)\end{array}$} \\
\hline Transformer & 0.0030 & 342.0 & 25.61 & 48,000 \\
Bus bar & 0.0017 & 24.0 & 365.0 & 500 \\
Breaker & 0.0036 & 83.1 & 105.4 & 12,000 \\
\hline
\end{tabular}


If two complemented minimal path-sets share basic events then the product is decomposed into a sum of two products. One sub-product is added to the list of products to be disjoint; the other sub-product (the one with the shared events only) is re-dealt with again (absorption and decomposition steps only). (Decomposition discipline).

The aforediscussed procedure is illustrated in the flow chart of Fig. 2.

\section{CPU and memory usage}

The implementation of this algorithm is based on a modular structure (i.e. using separate functions coded into black boxes). The same structure illustrated in the flow chart is used.

The order function of the initialization phase is $O(N$, $P)=P^{2}+\mathrm{NP}$.

where $P$ is the number of path-sets and $N$ is the number of variables.

For the product disjointing phase, the order function is $O(P)=P^{2 P-2}$. This is basically due to the decomposition discipline. In case no decomposition is required then $O(P)=P^{2}$

It is worth noticing that the order function of the product disjointing phase does not depend on the number of variable. This is due to the usage of bitwise property of $\mathrm{C} / \mathrm{C}++$.
Fig. 6 Reliability assessment results for single bus, double breaker-double bus and ring bus

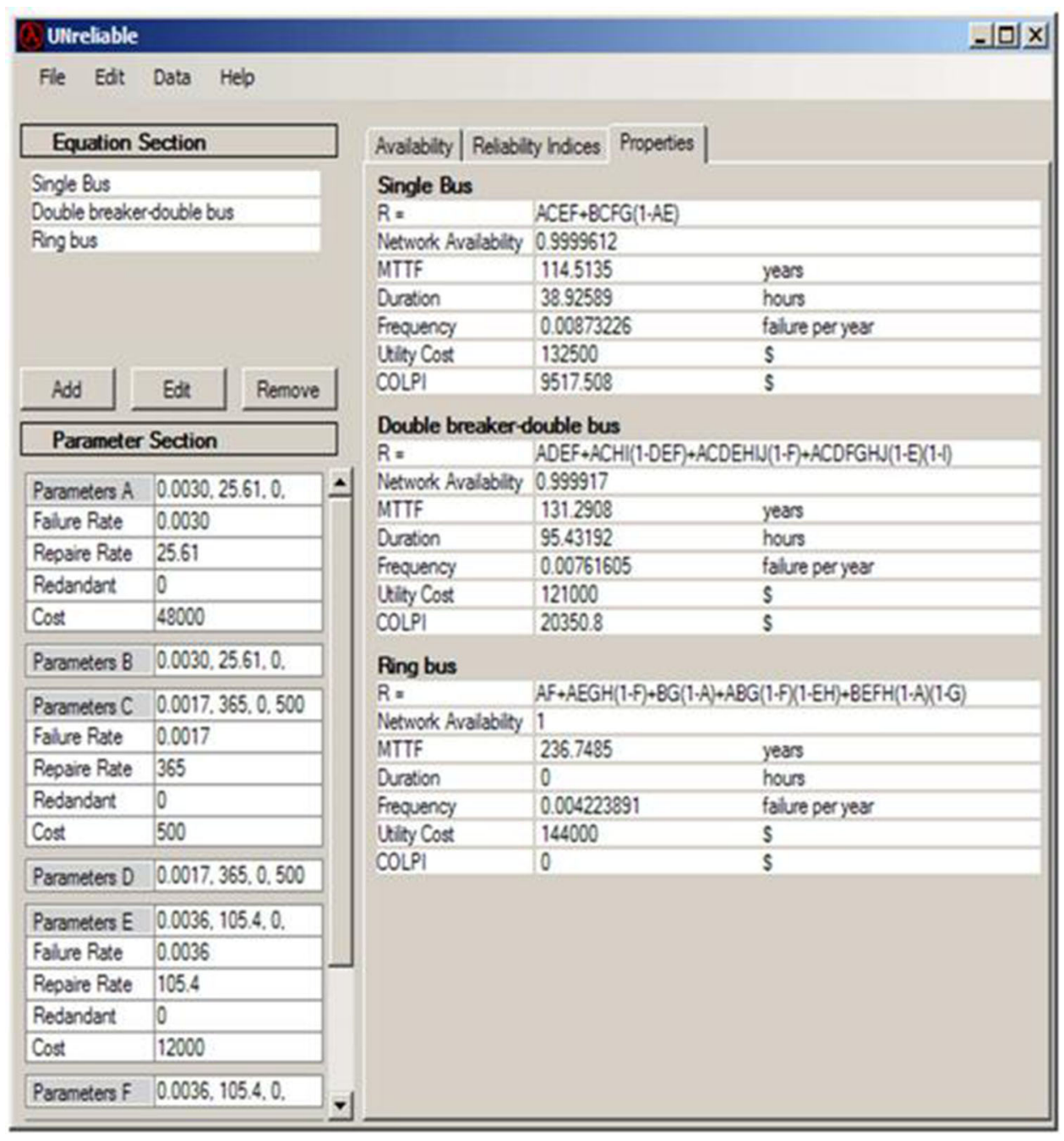

Table 2 Substation reliability indices

\begin{tabular}{lcclll}
\hline Configuration & Availability & MTTF $(\mathrm{y})$ & MTTR $(\mathrm{h})$ & Frequency $(\mathrm{fl} / \mathrm{y})$ & Total cost $(\$)$ \\
\hline Single bus & 0.9999612 & 14.5 & 38.92 & 0.008732 & 182,017 \\
Double breaker & 0.999917 & 131.3 & 95.43 & 0.007616 & 201,350 \\
Double bus ring bus & $\approx 1$ & 236.7 & $\approx 0$ & 0.004223 & 184,000 \\
\hline
\end{tabular}


Fig. 7 A breaker and a half substation configuration with labeled components

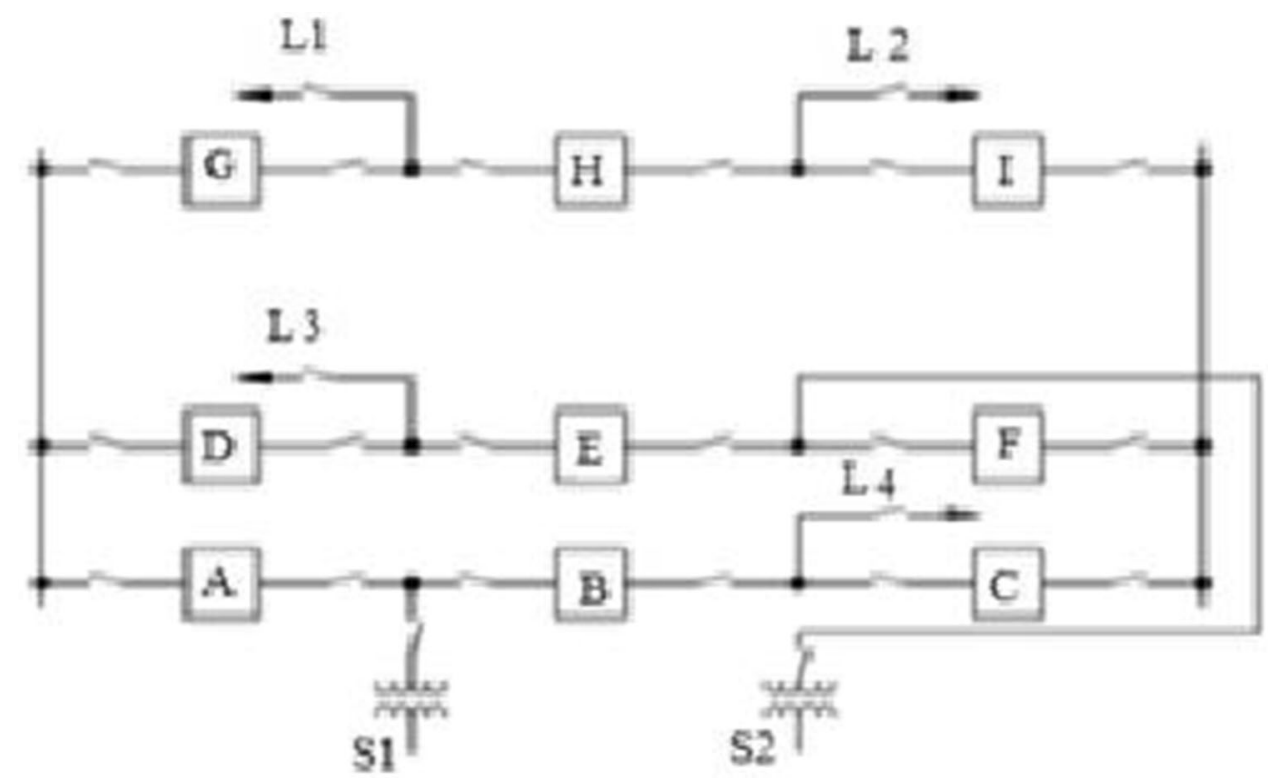

\begin{tabular}{|c|c|c|}
\hline Avalability & Reliabily Indces & Propeties \\
\hline \multicolumn{3}{|c|}{ breaker and a half } \\
\hline$R:$ & $+P^{2} z+2 P^{2}$ & $3-20^{\wedge} 5-20^{\wedge} 6+P^{\wedge} 7+2 P^{\wedge} 8 P^{\wedge} 9$ \\
\hline \multicolumn{3}{|c|}{ Network Avalability } \\
\hline MTTF & & years \\
\hline Duration & & hours \\
\hline Frequency & & falure peryear \\
\hline Witycosi & & $\$$ \\
\hline COLPI & & $\$$ \\
\hline
\end{tabular}

Fig. 8 Results of the disjoint algorithm on a breaker and a half

Concerning memory usage, a linked list structure is used to store and manipulate the equations. Combined with dynamic memory allocation, this structure avoids unnecessary usage of memory. For this particular algorithm, the required memory is: $P \times(4+N)$ bytes for a max of variables of $8, P \times(4+2 N)$ bytes for a max of 16 and $P \times(4+4 N)$ bytes for a max 32 variables. Plus enough space to hold input and output strings. The rest of the variables and buffers are local.

\section{Application to power distribution substations}

Three of the previously described configurations are analyzed (single bus, double breaker-double bus and ring bus). The components modeled are transformers, bus bars and breakers. These components are labeled in the following figures. For convenience, reclosers and source availability

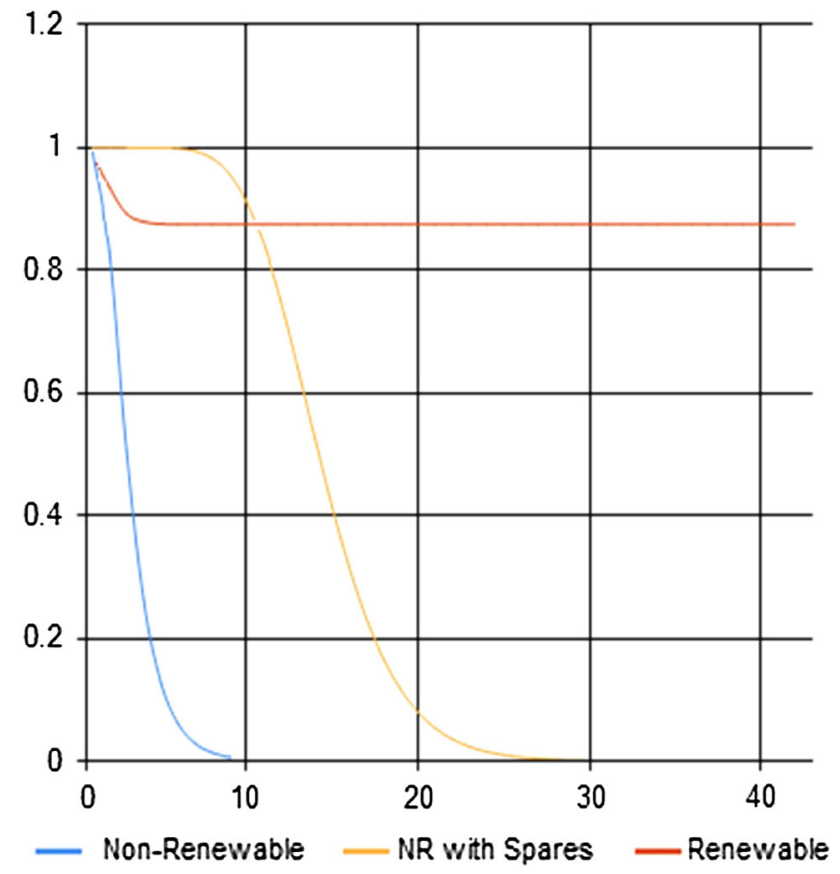

Fig. 9 Plot of a non-renewable (with and without spares) and a renewable behavior of breaker and a half substation

are assumed to be $100 \%$ reliable of current interest (Figs. 3, 4, 5).

It can be see that these configurations are symmetrical.

Therefore, output nodes have equivalent path-sets (i.e. the path-sets have equal availabilities and transition rates). For each configuration, one output is considered, and their path-sets are:

Single bus: AECF + BGCF. 


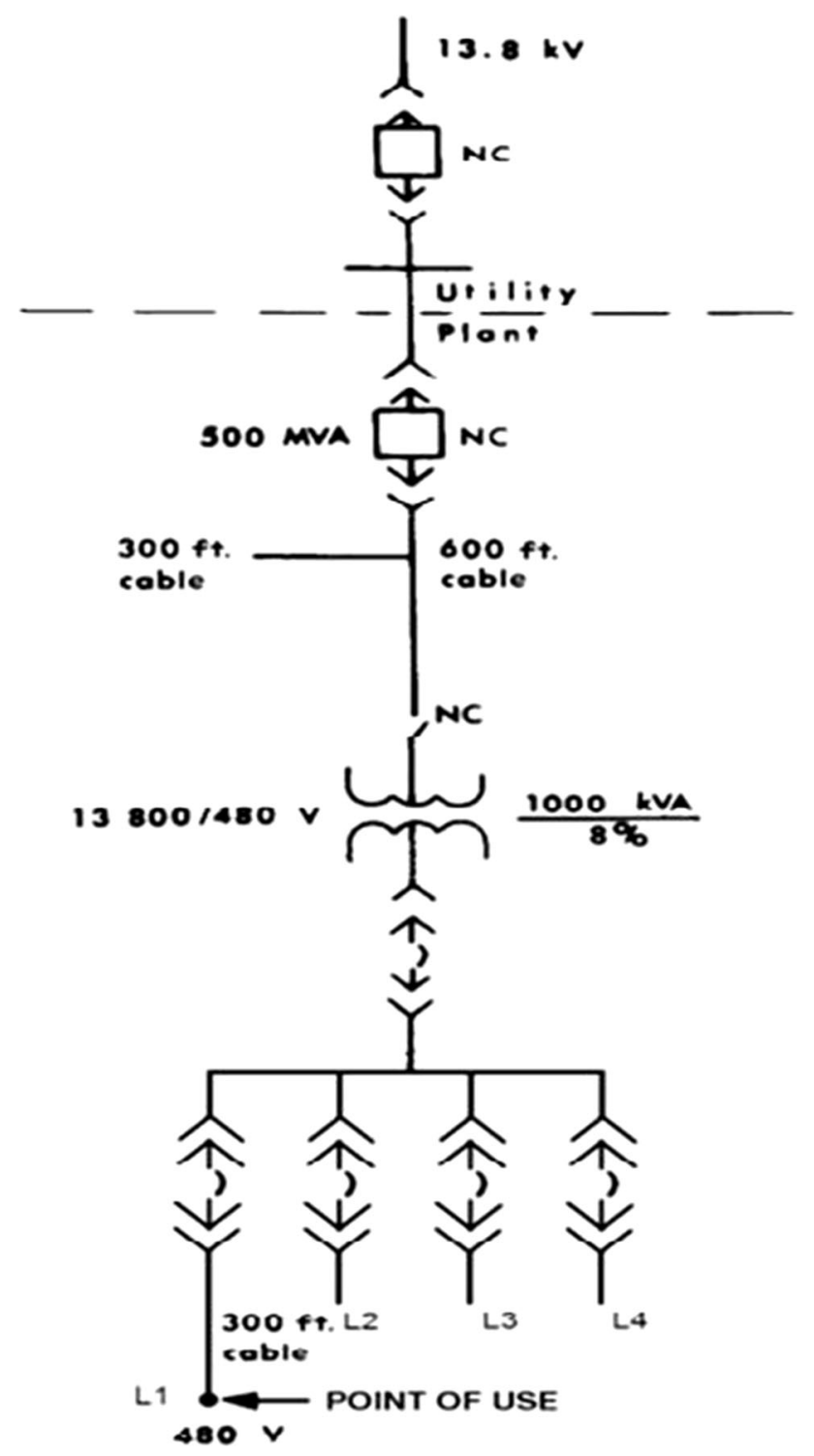

Fig. 10 Simple radial system - reliability and availability of power at $480 \mathrm{~V}$ (Singh and Billinton 1977)

Double breaker-double bus: $\mathrm{AFDE}+\mathrm{AICH}+$ AICHJDE + AFDJGCH.

Ring bus: $\mathrm{AF}+\mathrm{AEHG}+\mathrm{BG}+\mathrm{BHEF}$.

where $\mathrm{A}$ and $\mathrm{B}$ are transformers, $\mathrm{C}$ and $\mathrm{D}$ are bus bars and $\mathrm{E}, \mathrm{F}, \mathrm{G}, \mathrm{H}, \mathrm{I}$ and $\mathrm{J}$ are breakers.

The component reliability data and cost for this example are taken as in Table 1. Revenue lost per hour of substation downtime is $22,000 \$$. Average repair and start up cost per hour is $6,000 \$$, making it a total of $28,000 \$$ (Fig. 6).

\section{Comparison}

The results are tabulated in Table 2. Even though reclosers are discarded (assumed reliable), their cost needs to be considered. The total cost, then, is the summation of the utility cost (plus reclosers) and the cost of load point interruption COLPI (unserved power to customer and repair cost).

For a 5,000\$ recloser, total cost has a plus of $8 \times 5,000 \$, 12 \times 5,000 \$$ and $8 \times 5,000 \$$ for single bus configuration, double breaker-double bus and ring bus configuration, respectively.

Even with its well-known low reliability, the single bus exhibits an excellent availability level (4 nines). This is due to the reliable components composing this substation (small failure rates and small repair times).

The double breaker-double bus configuration has a slightly (but still 4 nines) lower availability than the single bus configuration. However, the mean time to failure (MTTF) as well as the failure frequency is less than that of the single bus configuration. The relatively high cost for this configuration disallows an unnecessary use of sophisticated configuration when made of reliable components. The ring bus configuration's availability is 1 but still has a non-zero failure frequency. This is due to the rounding-off of the very high availability for this configuration. Also, this latter has a large mean time to failure and almost zero repair time in case of total failure.

Non-renewable (with and without spares) and renewable substations

An example of three system behaviors is illustrated, using a breaker and a half topology. This behaviors' investigation is very important in the design phase, especially when the system is made for a specific mission time. The components' parameters, for this example, are chosen for convenience, and are not based on any actual survey.

In this example only breakers are considered (all of the other components are assumed to be perfectly reliable). The network and labels are illustrated in Fig. 7.

Path-set for load point L1 is:

From S1: AG + BCIH + ADEFIH + BCFEDG.

From S2: EDG + FIH + EDABCIH + FCBAG.

The resulting minimal path-set is: $\mathrm{AG}+\mathrm{EDG}+$ $\mathrm{FIH}+\mathrm{BCIH}$.

The reliability for non repairable components with spares is found using Poisons rule: (Chowdhury and Koval 2009; Dr Nahman 2002; Anderson 1998).

where $n$ is the number of spares.

The reliability polynomial is used to compute the availability of this substation (since all of the components are identical and have the same reliability $P$ ) for the different cases considered here (Fig. 8).

For the non-renewable mode, the breakers' failure rate is 0.36 failure/year. Then spares are used (4 spares). For the renewable case, the repair rate is considered to be 1 repair/ year. For all of these cases, mission time is 40 years. A graph of the three cases is illustrated in Fig. 9. 
Table 3 Transition rates and costs of radial system component (Anderson 1998)

\begin{tabular}{|c|c|c|c|c|c|}
\hline \multicolumn{2}{|c|}{ Component } & \multirow{2}{*}{$\frac{\lambda(\mathrm{fl} / \text { year })}{1.956}$} & \multirow{2}{*}{$\frac{\text { MTTR (h) }}{1.320}$} & \multirow{2}{*}{$\frac{\mu \text { (rp/year) }}{6,636}$} & \multirow[t]{2}{*}{ Cost $(\$)$} \\
\hline & $13.8 \mathrm{kV}$ power source from electric utility & & & & \\
\hline A & Protective relays (3) $13.8 \mathrm{kV}$ metalclad & 0.0006 & 5.0 & 1,752 & \\
\hline $\mathrm{B}$ & Circuit breaker Switchgear bus & 0.0036 & 83.1 & 105.4 & 40,000 \\
\hline $\mathrm{C}$ & Insulated (connected to 1 breaker) Cable $(13.8 \mathrm{kV}) ; 900$ & 0.0034 & 26.8 & 326.9 & \\
\hline $\mathrm{D}$ & $\mathrm{ft}$, conduit below ground & 0.0055 & 26.5 & 30.6 & 18,000 \\
\hline $\mathrm{E}$ & Cable terminations (6) at $13.8 \mathrm{Kv0}$ & 0.0018 & 25.0 & 350.4 & \\
\hline $\mathrm{F}$ & Disconnect switch (enclosed) & 0.0061 & 3.6 & 2,433 & \\
\hline G & Transformer $480 \mathrm{~V}$ metalclad & 0.0030 & 342.0 & 25.61 & 48,000 \\
\hline $\mathrm{H}$ & Circuit breaker Switchgear bus-bar & 0.0027 & 4.0 & 2,190 & \\
\hline I & (Connected to 7 breakers) $480 \mathrm{~V}$ metalclad & 0.0024 & 24.0 & 365.0 & 12,000 \\
\hline $\mathrm{J}$ & Circuit breaker $480 \mathrm{~V}$ metalclad circuit breakers (5) & 0.0027 & 4.0 & 2,190 & 5,000 \\
\hline $\mathrm{K}$ & (Failed while opening) & 0.0012 & 4.0 & 2,190 & 5,000 \\
\hline $\mathrm{L}$ & Cable $(480 \mathrm{~V}) ; 300 \mathrm{ft}$ conduit above ground & 0.0004 & 11.0 & 796.4 & 2,500 \\
\hline M & Cable terminations (2) at $480 \mathrm{~V} \mathrm{L1}$ & 0.0002 & 3.8 & 2,305 & \\
\hline $\mathrm{N}$ & Cable $(480 \mathrm{~V}) ; 300 \mathrm{ft}$ conduit above ground & 0.0005 & 11.0 & 796.4 & 3,000 \\
\hline $\mathrm{O}$ & Cable terminations (2) at $480 \mathrm{~V} \mathrm{~L} 2$ & 0.0002 & 3.8 & 2,305 & \\
\hline $\mathrm{P}$ & Cable $(480 \mathrm{~V}) ; 300 \mathrm{ft}$ conduit above ground & 0.0006 & 11.0 & 796.4 & 4,000 \\
\hline Q & Cable terminations (2) at $480 \mathrm{~V} \mathrm{~L} 3$ & 0.0002 & 3.8 & 2,305 & \\
\hline $\mathrm{R}$ & Cable $(480 \mathrm{~V}) ; 300 \mathrm{ft}$ conduit above ground & 0.0008 & 11.0 & 796.4 & 5,000 \\
\hline $\mathrm{S}$ & Cable terminations (2) at $480 \mathrm{~V} \mathrm{L4}$ & 0.0002 & 3.8 & 2,305 & \\
\hline
\end{tabular}

It is clear, from the graph that the renewable system has the highest availability. However, this is only true for a long run. For short mission systems, using spares provides higher reliability.

Simple radial distribution system

Many distribution systems are designed and constructed as single radial feeder systems, especially in rural areas. One simple radial system is shown in Fig. 10. It is used as an example to underline the different meaning of performance indices, as well as cases of loss of load (Chowdhury and Koval 2009).

The parameters of the components composing this network are provided in Table 3. It is normally found in practice that lines and cables have a failure rate which is approximately proportional to their length. Therefore, it is reasonable to assume higher failure rate for the other lateral distribution $(0.0005,0.0006$ and 0.0008 failure/year for load point 2, 3 and 4, respectively).

Since this network is radial then the path-set is directly deduced.

The four output nodes (L1, L2, L3 and L4) have 100, 80,70 and 50 customers, respectively. Figure 11 represents load profiles (power demands) for loads L1, L2, L3 and L4. The points worth noticing are the peaks during summer time and winter time. The source's power is $2.5 \mathrm{MW}$.

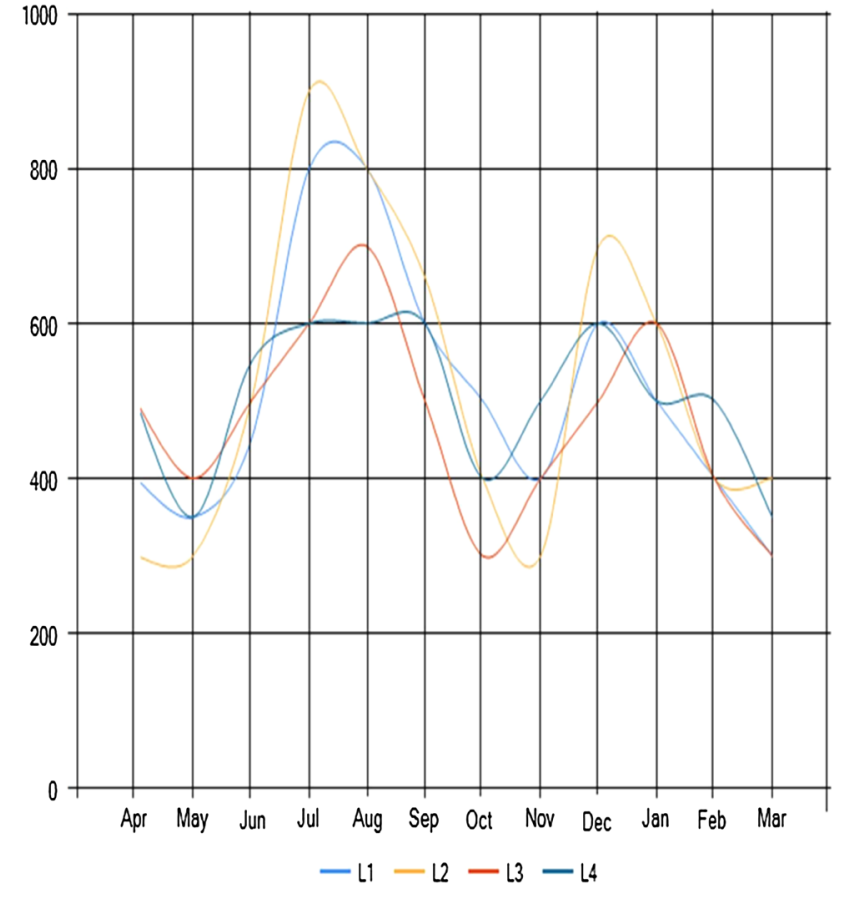

Fig. 11 Load profiles for loads $L 1, L 2, L 3$ and $L 4$

The results of this example are illustrated in Figs. 12, 13 , and 14.

This is a four load point availability graph, but only one is displayed. All of the graphs are superimposed; because 
their path-sets are highly reliable (see Fig. 12). The unavailability of electric power is mainly due to loss of load (blackout rolling not considered).

The following graph represents some commonly used indices, namely SAIDI, CAIDI, SAIFI and ASAI. The $y$ axis represents: percentage for ASAI, interruption/customer for SAIFI hours/customer for SAIDI and hours/ customer interruption for CAIDI.

The average system availability index (ASAI) is quite the same (if not exactly the same) as the load points' availabilities, which are consequences of the similarity between their individual availabilities.

SAIFI represents the average number of outages per customer per month. It reaches a maximum value of around 6 interruptions/customer between July and August. This is due to the drop of ASAI to $90 \%$.
Both SAIDI and CAIDI represent average interruption duration; nevertheless, their graphs exhibit different patterns. On one hand, CAIDI is the ratio of the total interruption duration over the total number of interruption. A decrease of the availability results in an increase of interruption duration and may cause an increase of the number of interruptions. As a consequence, the graph is bound between 15 and $19 \mathrm{~h} /$ customer interruption. On the other hand, SAIDI is the ratio of the total interruption duration over the total number of customers served. The denominator is constant resulting in a high dependability of this index to system availability. Figure 14 represents properties of the four load nodes. A slight decrease of their respective network availability (from L1 to L4) is to be noticed, along with an increase of failure frequency and cost of load point
Fig. 12 Availability of load power for points $L 1, L 2, L 3$ and L4

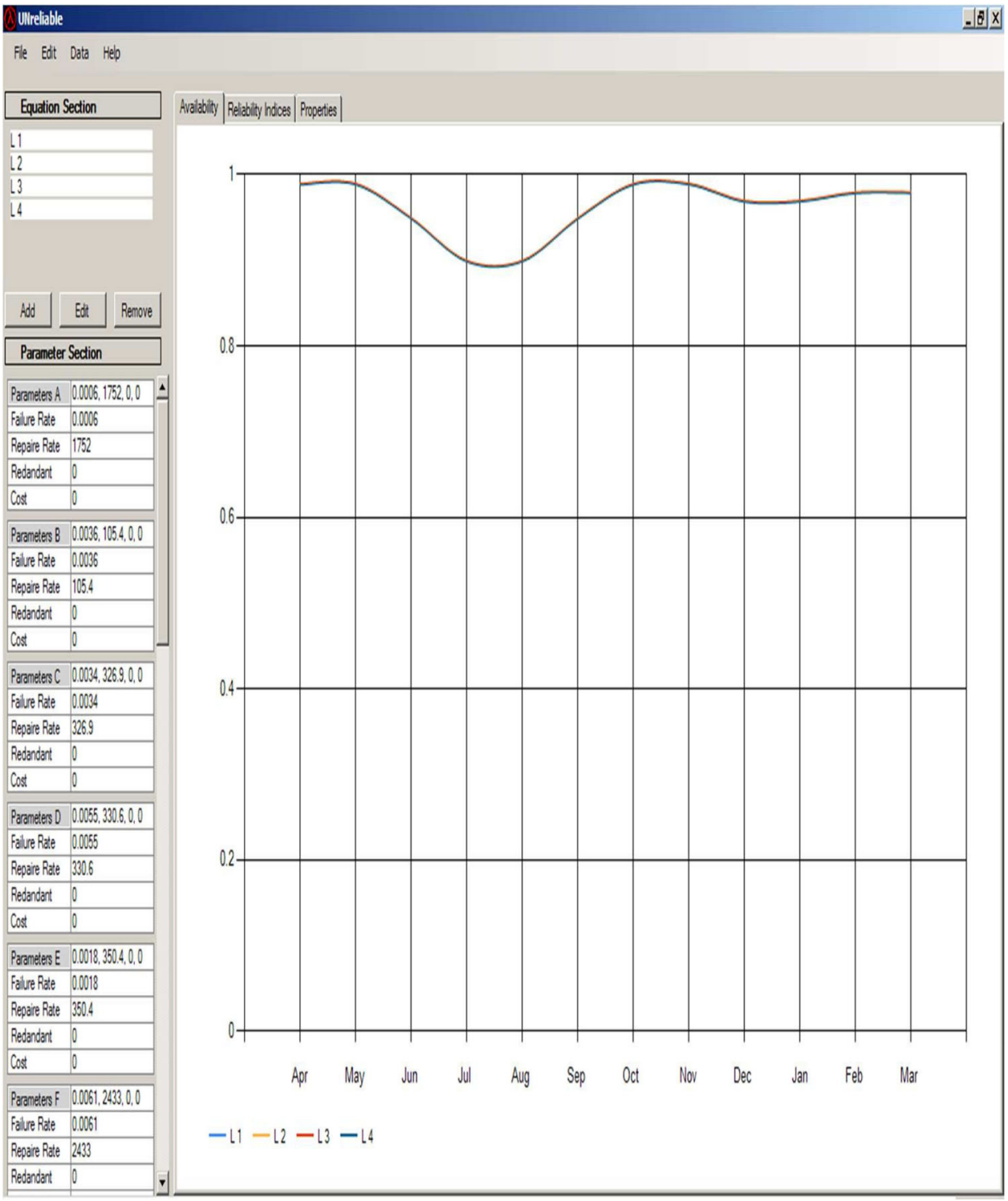


interruption. This is the result of the small increase of failure rates for longer lines and cables.

\section{Conclusion}

In this paper we can conclude the possibility of the investigation for the reliability expression and indices of different substation configurations on the use of the disjoint paths algorithm, because it reduces the order of the execution time to $2^{2 P-2}$ ( $P$ is the number of paths). While a direct approach or state space approach would result in an NP-hard problem. Since this approach is based on Boolean algebra (and probability theory), multiple state systems are unpractical. Only two state components are considered.

Power distribution systems and, more specifically, power distribution substations are built from two state components. Therefore, the aforementioned algorithm is perfectly suited for power distribution system reliability assessment. The application of this algorithm not only saves computational effort but, it uses also the path-set enumeration instead of the tedious state space enumeration.

While using this tool, component's transition rates need to be constants. Non-exponential component's transition rates are not considered (dependent events). However, surveys on power distribution system components report constant transition rates during the component's normal operating time. This encourages the use of this algorithm. In the case where power load and available power are considered, the system becomes a composition of a multistate subsystem and two state subsystems. A combination (if applicable) of state space approach and the presented algorithm can solve the problem.
Fig. 13 Performance indices CAIDI, SAIDI, SAIFI and ASAI of a radial system

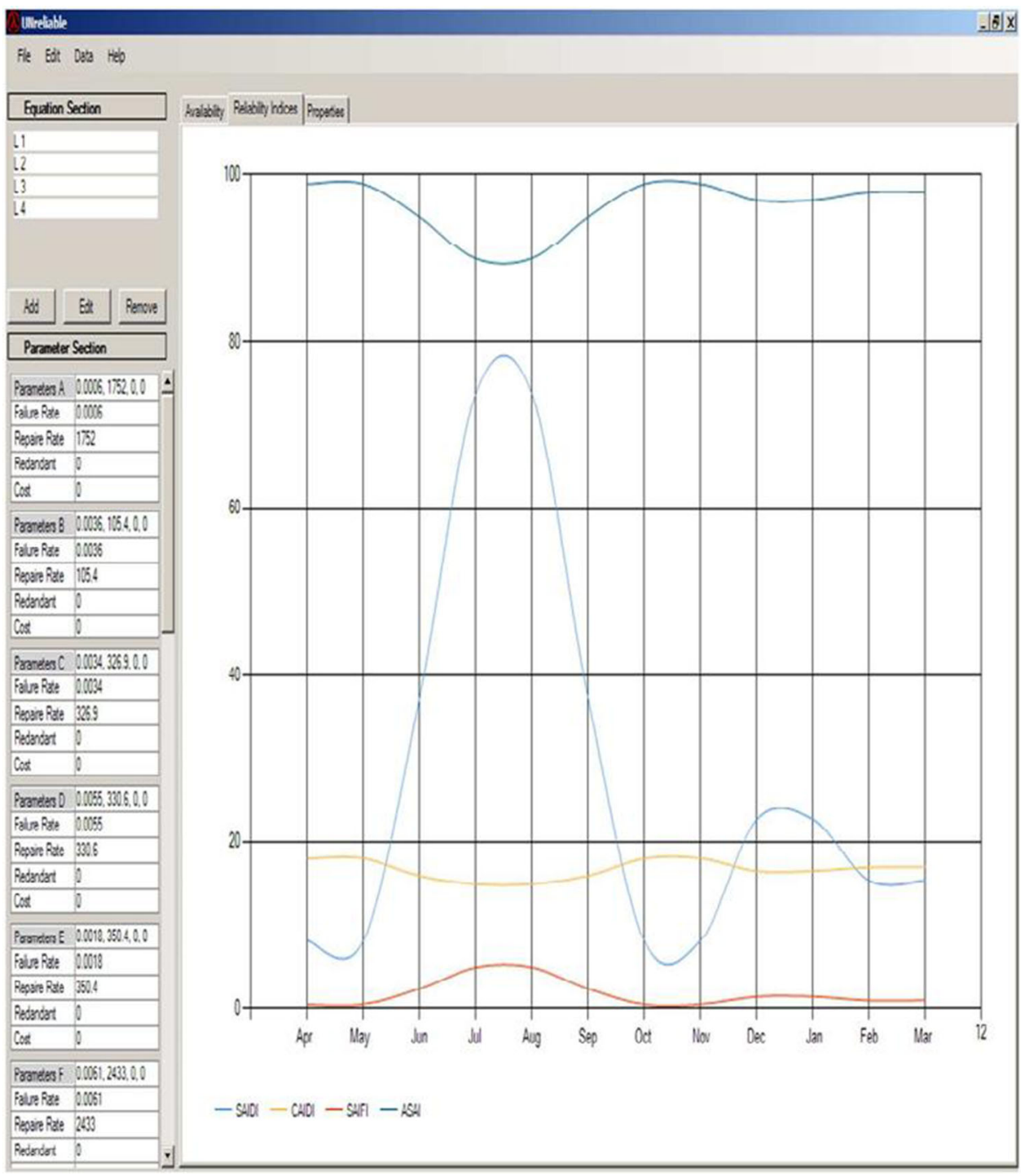




\begin{tabular}{|c|c|c|}
\hline \multicolumn{2}{|c|}{ Availability | Reliability Indices Properties } & \\
\hline \multicolumn{3}{|l|}{ L 1} \\
\hline$R=$ & \multicolumn{2}{|l|}{ ABCDEFGHIJKLM } \\
\hline Network Availability & \multicolumn{2}{|l|}{0.9998036} \\
\hline MTTF & 30.26469 & years \\
\hline Duration & 52.07882 & hours \\
\hline Frequency & 0.03303532 & falure per year \\
\hline Utility Cost & 142500 & $\$$ \\
\hline COLPI & 48172.33 & s \\
\hline \multicolumn{3}{|l|}{ L 2} \\
\hline$R=$ & \multicolumn{2}{|l|}{ ABCDEFGHIJKNO } \\
\hline Network Availabilty & \multicolumn{2}{|l|}{0.9998034} \\
\hline MTTF & 30.17638 & years \\
\hline Duration & 51.97415 & hours \\
\hline Frequency & 0.03313198 & failure per year \\
\hline Utility Cost & 142500 & $s$ \\
\hline COLPI & 48216.19 & \$ \\
\hline \multicolumn{3}{|l|}{ L3 3} \\
\hline$R=$ & \multicolumn{2}{|l|}{ ABCDEFGHIJKPQ } \\
\hline Network Availability & \multicolumn{2}{|l|}{0.9998033} \\
\hline MTTF & 30.0886 & years \\
\hline Duration & 51.85439 & hours \\
\hline Frequency & 0.03322864 & fallure per year \\
\hline Utilty Cost & 142500 & $s$ \\
\hline COLPI & 48245.43 & $\$$ \\
\hline \multicolumn{3}{|l|}{ L 4} \\
\hline$R=$ & \multicolumn{2}{|l|}{ ABCDEFGHINKRS } \\
\hline Network Availability & \multicolumn{2}{|l|}{0.9998031} \\
\hline MTTF & 29.91457 & years \\
\hline Duration & 51.61697 & hours \\
\hline Frequency & 0.03342194 & failure per year \\
\hline Utility Cost & 142500 & $\mathrm{~S}$ \\
\hline COLPI & 48303.91 & $\$$ \\
\hline
\end{tabular}

Fig. 14 Property tab for the four load point $(L 1, L 2, L 3$ and $L 4)$

As a future work, components with non-exponential distributions could be considered. Approximated models for these components and technique for approximation (such as supplementary variable method) should be investigated.

Other repair modes should be considered. Renewable components and the use of spares are not the only way to handle failure. Many widely used strategies need to be considered such as the use of standby units (cold or warm) or scheduled maintenance.

Conclusions about different configurations are stated in the comparison section of this paper.

Open Access This article is distributed under the terms of the Creative Commons Attribution License which permits any use, distribution, and reproduction in any medium, provided the original author(s) and the source are credited.

\section{References}

Anderson PM (1998) Power system protection. Volume 4 of IEEE press series on power engineering. Wiley, USA

Bashiri M, Karimi H (2012) Effective heuristics and meta-heuristics for the quadratic assignment problem with tuned parameters and analytical comparisons. J Ind Eng Int 8(1):1-9

Beheshti Z (2013) A review of population-based meta-heuristic algorithms. Int J Adv Soft Comput Appl 5(1):1-35

Billinton R, Allan RN (1996) Reliability evaluation of power systems, 2nd edn. University of Saskatchewan, Canada Editor Plenum, New York

Brown Richard E (2002) Electric power distribution reliability. ABB Inc., Raleigh

Chowdhury AA, Koval DO (2009) Power distribution system reliability: practical methods and applications, Institute of Electrical and Electronics Engineering.inc. Wiley, Hoboken

Dote Y, Ovaska SJ (2001) Senior member, IEEE, industrial applications of soft computing: a review. Proc IEEE 89(9):1243-1265

Hale PS, Arno RG, Koval DO (2001) Analysis techniques for electrical and mechanical power systems. In: Proc. 2001 IEEE I\&CPS Tech. Conf., pp 61-65

IEEE Std 493-1997 (Revision of IEEE Std 493-1990) (1998) IEEE recommended practice for the design of reliable industrial and commercial power systems

Koval DO, Jiao L, Arno RG, Hale PS (2002) Zone-branch reliability methodology applied to Gold Book standard network. IEEE Trans Ind Applicat 38:990-995

Nahman JM (2002) Dependability of engineering systems: modelling and evaluation. University of Belgrade, Faculty of electrical engineering, Springer, Heidelberg

Singh C, Billinton R (1977) System reliability modelling and evaluation. Hutchinson Educational, London

Wang W, Loman JM (2002) Application of the minimal cut set reliability analysis methodology applied to the Gold Book standard network. In: Proc. 2002 IEEE I\&CPS Tech. Conf., pp 82-93

Xing J (2012) A simple algorithm for sum of disjoint products. Beijing Institute of Technology IEEE, Beijing

A. Bourezg was born on March 1974. He was graduated as engineer in electronics and electrical engineering in 1997. He joined the Federal Polytechnic School of Lausanne (Switzerland) for postgraduate courses in electrical engineering. He got his magister from Oil and Chemistry Faculty, University of Boumerdes in 2007.Currently, lecturer in Electronics and Electrical Engineering Institute; University of Boumerdes-Algeria.

H. Meglouli Professor in Automation of Industrial Processes Department of Oil and Chemistry Faculty_University of Boumerdes. 